\section{Mimicking MacGyver in the Oncology Clinic: Improvisation and Creative Solutions, Only Rarely Involving Duct Tape}

\author{
David P. Steensma, MD
}

Among the most popular television series in the late 1980s was MacGyver, an $\mathrm{ABC}$ action drama that followed the adventures of gun-averse secret agent Angus MacGyver, played by a spectacularly mulleted Richard Dean Anderson. The show's central gimmick was Agent MacGyver's extraordinary ability to improvise clever mechanical solutions to life-and-death crises-spur-of-the-moment fixes that often relied on duct tape or a Swiss Army knife. Whether he was bypassing a blown fuse with foil from a chewing gum wrapper, using baked potatoes as projectile weapons, or, less plausibly, enlivening a casket with an outboard motor, MacGyver was never dull.

As a testament to how MacGyver's capacity for seemingly impossible kludges became part of popular culture, Saturday Night Live ran a series of "MacGruber" parody sketches, which later morphed into a full-length, fully forgettable feature film. Television presenters continue to mention "MacGyverisms" more than 20 years after the series' cancellation, and MacGyver's brainchildren are still put to the test on the popular science program MythBusters.

My own first consciously MacGyveresque moment was prompted by an automotive crisis along Michigan's Route 131 during my impoverished student days, when the original episodes were still airing. An overheated Volkswagen led me to try a trick I'd heard discussed by Click and Clack on National Public Radio's Car Talk: patching a leaking radiator by cracking an egg into it. (The egg boils, the white congeals, and the gloopy mess seals the leak.)

The egg trick is a temporary solution, if it works at all—and it didn't for me. But thinking of this incident led me to wonder what MacGyver might be able to teach busy doctors now. He might not have much to teach in the way of mechanical jerry-rigging, since a day in the clinic rarely requires clinicians to develop radiographic film with orange juice, or distract villainous prior authorization reviewers with a camera flash. But medical practice in this era of dysfunctional or failed systems - when institutions assume physicians have unlimited time to perform clerical and administrative tasks, pharmaceutical firms charge prices for new drugs that resemble Scott Boras-negotiated athletic contracts, and insurance companies seem to constantly push the bounds of what they can get away with-demands versatility and creative solutions. Clinical practice in the United States in the 21st century has become a long row to hoe. If ever there was an unstable edifice requiring MacGyver-like creativity to prop it up, caring for sick patients today is just such a wobbly structure.

Although leatherman multitools may be in short supply in clinic workrooms, MacGyverish behavior is not difficult to find. Frustration and desperation breed invention, and I am endlessly amazed by the ability of my colleagues to improvise practices that may not fit into a bureaucrat's tick box but are necessary to obtain appropriate care for patients who might benefit. Creative ICD-9 coding, using a drug for purposes for which it is effective but was not originally intended, or making sure scheduling personnel hear the magic combination of words to get a needy patient onto a consultation list or procedural calendar are daily necessities. Within the bounds of the law, we do what we need to do to provide good care for our patients. Clinicians may indulge in deep thinking and theorizing after-hours, but in the clinic and on the wards, we are usually forced to be pragmatists.

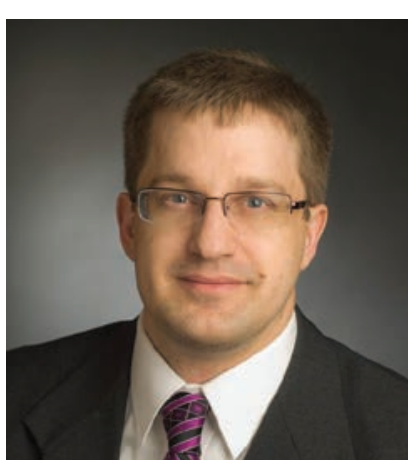

David P. Steensma, MD

David P. Steensma, MD, is Associate Professor of Medicine at Harvard Medical School; consultant for the Adult Leukemia Group, Department of Hematological Malignancies at Dana-Farber Cancer Institute; and attending physician at Brigham \& Women's Hospital in Boston, Massachusetts.

Dr. Steensma is on the data safety monitoring committee at Amgen is chair of the MDS registry and on the advisory board for Celgene; is on the advisory board for Incyte; and provides consulting for MEI Pharma. He reports no other relevant disclosures.
The ideas and viewpoints expressed in this commentary are those of the author and do not necessarily represent any policy, position, or program of NCCN. 
Another example, this time from the Star Trek canon-while somewhat afield from cancer care- -is inspiring when it comes to cutting Gordian knots. Young James Kirk, while still a cadet at Starfleet Academy, faced an impossible test as part of his training: a simulated rescue of a civilian vessel, the Kobayashi Maru, which sent out a distress beacon after becoming disabled by a mine in a Klingon-controlled space zone. Cadets who tried to rescue the vessel violated the Klingon Neutral Zone and provoked war with the enemy, while those who ignored the catastrophe or delayed the response in order to negotiate caused the vessel's crew and passengers to die. No student had ever solved this conundrum, because it was designed to be a "no-win" scenario. However, much to the annoyance of a by-the-book Academy instructor named Spock, Cadet Kirk secretly reprogrammed the simulator, disabling the Klingon response and allowing the stranded ship to be rescued without a reprisal. When criticized for not playing by the rules, Cadet Kirk said he did not believe in the reality of no-win scenarios, and the future USS Enterprise captain was praised by his teachers for his original thinking.

This is a utilitarian ethic, to be sure, and it must not be taken too far. But when rules that are meant to protect patients or health care teams inadvertently do the opposite, when guidelines are arbitrary and capriciously applied, or when a system is one in which competing goals are impossible to reconcile, sometimes you cannot stand on ceremony. Imagine a scenario in which a hospitalized patient needs a PICC line inserted before she can go home, but the interventional radiology department won't insert it for 48 hours, because the on-call resident ordered a blood culture for a low-grade fever during the night, and the PICC team's policy is that all cultures must be negative for 48 hours before a line can be placed. (This scenario is not difficult for me to visualize, since it happened to me twice recently.) You might wait 48 hours for the culture to be negative and follow hospital protocol to the letter; after all, this rule was put in place for a good reason, to decrease the likelihood of infection. But you might also be tempted to call the microbiology laboratory and cancel the culture, erasing its existence in the computer so that it won't be seen by the PICC teamespecially if that fever was almost certainly a tumor fever anyway, like the culturenegative fevers the patient had each of the previous 3 nights.

The remarkable nurse practitioner who is the glue that holds my institution's clinical leukemia program together carries a thick black book whenever she is at work. Instead of a list of personal contacts, her black book catalogues work-arounds and reminders of how to get things done at our institution. These effective techniques, which she has learned from long experience on the front lines, include special codes for getting blood drawn for research studies, the correct place to send esoteric tests and how to label them (since an order for an unusual evaluation can strike the laboratory sample control worker as something never heard of, and potentially create a similar commotion to getting in line at Subway and ordering a tortilla with ostrich breast and Gruyère), and critical telephone numbers for each department or scheduling areaoften a direct link to the best person in that area with the knowledge and care to get the job done right.

As a naïve new attending physician, I once sent a patient with recurrent leukemia and a malignant pericardial effusion to the cardiology clinic without consulting the nurse practitioner first, only to have the patient return more breathless than before, the effusion unaddressed, with a consult note in her chart recommending atorvastatin because of a high low-density lipoprotein cholesterol level. I learned then that my colleagues only ever sent their patients to a subset of cardiologists who are clinically savvy and have a special interest in seeing oncology patients. So now I have an electronic black book of my own, and it is enormously helpful in delivering the type of "special sauce" care that makes patients and their families so grateful — though I still ask the nurse practitioner for advice whenever I encounter a blank page. 
Nurses are the ultimate wizards of creative solutions. If you want to see inventiveness (and acrobatics worthy of Cirque du Soleil), watch an experienced nurse take blood from a central venous catheter that is not working well. An oncology nurse who has been around the block a few times can solve both equipment and social problems that would bedevil a team of doctors. And going the extra mile for patients seems to be in their blood.

One would think that after 15 years of working within a relatively narrow area of oncology (and with an ever-expanding digital black book) that I would have developed a range of techniques for getting things done that are as second nature as driving a car, and that I would be able to accomplish my daily kludgework without thinking and would rarely find myself on an unfamiliar dead-end street. But clinical medicine is nothing if not a continuing education, and the thousands of potential combinations of diseases, treatments, insurance rules, institutional policies, patient personalities, and social situations result in endless variations. Guidelines can provide a useful map, especially through those back roads of medicine where we travel less frequently, but they can be of limited help when we need to get our hands dirty with real patients in difficult situations.

And the rules, like the guidelines, keep changing. A hemoglobin target that was fine for dosing Mrs. Murphy's darbepoetin last week is now no longer acceptable, for some non-biology-based reason known only to her $\mathrm{HMO}$ administrator. Meanwhile, Mr. Smith was doing fine on dasatinib, meeting all the NCCN-defined treatment milestones for chronic myeloid leukemia therapy, but now his insurer is demanding he switch to imatinib, because it is cheaper. And the ornate workaround required to get Mr. Jones his lenalidomide via the crummy blue-light-special plan he signed up for in his state's health insurance exchange is now moot because he's become eligible for Medicare.

An afternoon in the frenzied oncology clinic facing evolving challenges like these is a long way from Starfleet Academy or that lazy summer afternoon at the lake when, as an 8-year-old boy desperate to catch a fish for dinner with a hook and fishing line but no bait, I reeled in a perch on a hook that I'd fitted with a piece of grass smeared with suntan lotion. But a similar degree of originality and desperate innovation are often required to deliver medical care of the sort that doesn't make its way into guidelines. The "right" way to get it done, as Angus MacGyver would surely agree, is the way that works. 